\title{
Two-dimensional nonlinear time fractional reaction- diffusion equation in application to sub-diffusion process of the multicomponent fluid in porous media
}

\author{
P. Pandey $•$ S. Das $\cdot$ E-M. Craciun $\cdot$ T. Sadowski 10
}

Received: 7 May 2020/ Accepted: 29 October 2020/Published online: 20 November 2020

(C) The Author(s) 2020

\begin{abstract}
In the present article, an efficient operational matrix based on the famous Laguerre polynomials is applied for the numerical solution of twodimensional non-linear time fractional order reactiondiffusion equation. An operational matrix is constructed for fractional order differentiation and this operational matrix converts our proposed model into a system of non-linear algebraic equations through collocation which can be solved by using the Newton Iteration method. Assuming the surface layers are thermodynamically variant under some specified conditions, many insights and properties are deduced e.g., nonlocal diffusion equations and mass conservation of the binary species which are relevant to many
\end{abstract}

P. Pandey $\cdot$ S. Das

Department of Mathematical Sciences, Indian Institute of

Technology (BHU), Varanasi 221005, India

e-mail: ppunique1133@gmail.com

S. Das

e-mail: sdas.apm@iitbhu.ac.in

\section{E-M. Craciun}

Faculty of Mechanical, Industrial and Maritime Engineering, Ovidius University of Constanta Bd.

Mamaia 124, 900527 Constanta, Romania

e-mail: mcraciun@univ-ovidius.ro

T. Sadowski $(\bowtie)$

Department of Solid Mechanics, Faculty of Civil Engineering and Architecture, Lublin University of Technology, 20-618 Lublin, Poland

e-mail: t.sadowski@pollub.pl engineering and physical problems. The salient features of present manuscript are finding the convergence analysis of the proposed scheme and also the validation and the exhibitions of effectiveness of the method using the order of convergence through the error analysis between the numerical solutions applying the proposed method and the analytical results for two existing problems. The prominent feature of the present article is the graphical presentations of the effect of reaction term on the behavior of solute profile of the considered model for different particular cases.

Keywords Fractional calculus - Mass conservation . Two-dimensional reaction-diffusion equation . Convergence analysis · Laguerre polynomial

\section{Introduction}

It is not justified to categorize the fractional calculus theory as a young science. The origin of fractional calculus is as old as classical calculus itself. During the past few decades it has become the focus of interest of many disciplines of science and technology which provides an excellent and efficient tool for modeling and describing various scientific and complex engineering phenomena such as aerodynamics, polymer rheology, electrodynamics of complex medium, fluiddynamic traffic model $[23,33,34,44,56]$ etc. In the 
last few decades there have been a lot of research on the applications of fractional calculus theory to various scientific fields ranging from the physics of diffusion and advection phenomena to control system of finance and economics. Fractional differentiation has a lot of advantages on the simulation of dynamical systems and physical phenomena as compared to integer order differentiation, due to its non-Markovian and non-local behaviors. In many cases it is not possible to model the known equations in fractional order forms. For this some basic physical postulates are to be satisfied before giving its shape into fractional order system. Therefore every equation can not be generalised simply by replacing the integer order derivative by fractional order derivative.

From literature survey, it is seen that the fundamental ideas of the algorithms correlated to the ideas proposed by authors of $[3,57]$ and Bhrawy [6] have been used to develop the efficient and accurate algorithms for the purpose of solving partial differential equations [32]. Additionally, for finding the numerical solutions of linear fractional differential equations (FDEs), Bhrawy et al. [7] have developed an operational matrix with the popular Laguerre polynomials for the fractional order integration and have revised the generalized Laguerre polynomials on the semi-infinite intervals. In [1], the authors have introduced an operational matrix for the fractional order derivative for solving the linear and non-linear FDEs with given initial conditions.

A significant portion of fractional calculus theory is the two-dimensional fractional differential equations and integral equations which have further been studied in the articles [43, 45-48]. In [12], the authors have solved the two-dimensional fractional percolation equation in a non-homogeneous porous med- ium. In the present article, an accurate and efficient numerical technique has been proposed to solve two-dimensional fractional order reaction-diffusion equation arising in porous media.

Several kinds of flow problems like percolation flow problems, seepage flow problems have been examined and discussed in many aspects of research fields including groundwater hydraulics, seepage hydraulics, fluid dynamics and groundwater dynamics in the porous media $[13,26]$. The two-dimensional flow of the solute in the porous medium is governed under the hypothesis of continuity and Darcy's law is given by J. Bear and A. Verruijt [4] in the equation

$$
\frac{\partial}{\partial x}\left(k_{x} \frac{\partial P}{\partial x}\right)+\frac{\partial}{\partial y}\left(k_{y} \frac{\partial P}{\partial y}\right)=A_{0} \frac{\partial P}{\partial t},(x, y) \in \Omega
$$

where $A_{0}$ denotes the specific storativity, which is approximated by the effective porosity or specific yield; $P$ denotes the pressure head, the intrinsic permeability of the medium is given by $K=\frac{k \rho g}{\mu}$, which is the hydraulic conductivity having the components in the $x, y$ directions as $k_{x}$ and $k_{y} ; \rho$ denotes the water density ; $\mu$ be the viscosity; and $g$ the gravitational acceleration; $\Omega$ is the percolation domain.

Since there is a large digression from the ordinary Gaussian diffusion, therefore the underlying superposition is not appropriate in tracing the movement of the solute concentration in a porous medium which is nonhomogeneous. Additionally, a supplementary realistic model can be obtained by the consideration of the application of fractional order density gradient to recapture the non-homogeneity of the porous medium. The Modified Darcy law was proposed by the authors of [22] with Riemann-Liouville fractional order derivative. Under the assumption of the continuity of seepage flow and the Darcy law, the percolation equation (1) is deduced into the the following forms as these assumptions are not valid for real seepage flow. The differential system with ordinary derivative does not quantify the flow of fluid with spatial path dependency and/or time memory. This limitation in the application of ordinary differential system has motivated the authors of [22] to model the fractional Darcy law.

$$
\begin{aligned}
& q_{x}=k_{x} \frac{\partial^{\alpha_{1}} P}{\partial x^{\alpha_{1}}}, \quad 0<\alpha_{1}<1, \\
& q_{y}=k_{y} \frac{\partial^{\alpha_{2}} P}{\partial y^{\alpha_{2}}}, \quad 0<\alpha_{2}<1 .
\end{aligned}
$$

In the special case, the usual Darcy law can be obtained by putting $\alpha_{1}=\alpha_{2}=1$.

Einstein's theory of Brownian motion reveals that the mean square displacement (MSD) of a particle moving randomly is proportional to time, which also can be justified for the case of a simple integer order linear diffusion equation. But as the research on fractional calculus progresses, it is found that the MSD for the anomalous case i.e., for time fractional diffusion equation, increases slowly with time. For the linear time fractional diffusion equation $\frac{\partial^{\alpha} u}{\partial t^{\alpha}}=\frac{\partial^{2} u}{\partial x^{2}}$, 
the MSD is $\left\langle X^{2}(t)\right\rangle \sim t^{\alpha}$, where $0<\alpha<1$ is the anomalous diffusion exponent. The equation represents an evolution equation which generates the fractional Brownian motion, a generalization of Brownian motion. Thus it is seen that for the diffusion model, if the integer order time derivative is replaced by the fractional order time derivative, it changes the fundamental concept of evolution of foundation of physics. The physical meaning of the fractional order time derivative related to the statistics is the waiting time in accordance with the Montroll-Weiss theory. Hilfer and Anton [25] have showed that MontrollWeiss continuous time random walk (CTRW) with a Mittag-Leffler waiting time density is equivalent to a fractional order master equation. Later, Hilfer [24] explained that this underlying CTRW of the model is connected to the time fractional diffusion equation in the asymptotic sense of long time and large distance. Thus random walk approach is needed to simulate diffusive phenomena of a fractional order equation. Gorenflo et al. [18] stated that the time fractional order diffusion equation generates a class of symmetric densities whose moments of order $2 m$ are proportional to the $m \alpha$ power of time. Thus classes of nonMarkovian stochastic processes can be obtained, which exhibit slow anomalous diffusions. By using fractional order Fokker-Plank equation approach, Metzler et al. [35] have shown that anomalous diffusion is based upon Boltzman statistics. Many researchers have used fractional equations during description of Levy flights or diverging diffusion. The tool is very powerful in modeling multi scale problems, characterized by wide time or length scale. The fractional order differential operator has the characteristic of non-local property, which states that the future state not only depends upon the present state but also upon all of the history of its previous states. Due to the greater flexibilities, the fractional order models have gained popularity to investigate the dynamical system.

The anomalous diffusion process seeks the attention of many researchers [5, 8, 14]. Anomalous diffusion can be easily seen within complex systems like diffusion process in porous media. The fluid particles undergoes in sub-diffusion process if $\alpha<1$. A subclass of anomalous diffusive system is the fractional sub-diffusion equation (FSDE), which can be illustrated from the standard parabolic PDE by replacing the first-order time derivative with a fractional derivative of order $\alpha, 0<\alpha<1$ (Moodie and Tait [37]).

In the real world problems, the FSDE has been widely applied in several fields of research $[11,20,30]$. So finding the solutions of these equations have become increasingly interesting and popular. The authors of [17, 36, 39] have provided a typical explanation of the anomalous diffusion process based on the continuous time random walk. The process of anomalous diffusion for real complex physical systems can be well explained by fractional order diffusion models.

The variations of the solute concentration with respect to the column length and time depend upon the several species and factors in fluid. It is very hard to find the overshoots of probability density function for the several cases like conservative and non-conservative systems. In addition to this, interactions of the solutes with medium or other species play a significant role in the flow of the fluid through porous media. In order to analyze the diffusion of the solute in porous media in two-dimension, an attempt has taken in the present article to develop a two-dimensional subdiffusion physical model (4) with the prescribed initial and boundary conditions (5-9), where the fractional derivative is taken in Caputo sense. The fractional time derivative corresponds to long-time heavy tail decay.

$$
\begin{array}{r}
\frac{\partial^{\alpha} u}{\partial t^{\alpha}}=\frac{\partial}{\partial x}\left(u \frac{\partial u}{\partial x}\right)+\frac{\partial}{\partial y}\left(u \frac{\partial u}{\partial y}\right)+k u(1-u) ; 0<\alpha<1, \\
0 \leq x \leq 1,0 \leq y \leq 1,
\end{array}
$$

with

$$
\begin{aligned}
& u(x, y, 0)=0, \\
& u(0, y, t)=c_{0}, \\
& u_{x}(1, y, t)=0, \\
& u_{y}(x, 0, t)=0, \\
& u_{y}(x, 1, t)=0,
\end{aligned}
$$


where $\alpha$ is an arbitrary fractional order, $k$ denotes the reaction coefficient and $c_{0}$ be a constant. For the approximation of solute concentration we have used Kronecker product which is discussed later.

When the ground water is mostly adulterate, then the resuscitate is considered to be very difficult and more expensive. A very careful approach and attention is very much necessary for describing the boundary conditions, problem domain and model parameters for using the numerical approach of groundwater model of the field problems. Hydrology is an interdisciplinary part of science and engineering, in which the topic of solute transport through the groundwater is included.

The generalized solute transport model in the porous media is the well known reaction-advectiondispersion equation (RADE) [15, 27]. This equation has the combined effects of advection, reaction and dispersion process due to which the concentration of the solute is dispersed while transported down along the stream and sometimes it reacts with the medium through which it moves. From the mass balance principles, the advection-dispersion equation can be easily derived. The modeling of solute transport is very helpful for the prediction of solute concentration in rivers, streams, aquifers and lakes, which can be represented mathematically as

$$
\frac{\partial u}{\partial t}=\nabla \cdot(d \nabla u)-\nabla(v u)+R,
$$

where $R$ denotes the reaction term of the species $u$, and the symbol $\nabla$ is defined in $R^{n}$ in terms of partial derivative operators as $\nabla=\left(\frac{\partial}{\partial x_{1}}, \ldots, \frac{\partial}{\partial x_{n}}\right)$.

In the right-hand side of the equation (10), the first term describes the dispersion phenomena, the second term represents the advection process and the last term is for the reaction kinetics. If there is no reaction between the solute and the medium through which the solute moves, and also there is no kind of radioactive decay then this type of system is called as conservative system, otherwise it is non-conservative. In the case of non-conservative system, the last term of the equation (10) is encountered. If there is only diffusion process which is responsible for the movement of the solute concentration, then the above equation is known as diffusion equation.

In this article a drive has been taken to develop the operational matrix with Laguerre polynomials to use in collocation method to find the numerical solution of two-dimensional fractional order non-linear reactiondiffusion equation. We have introduced an efficient way to approximate the non-linear multiple-order fractional boundary value problem with the help of Laguerre spectral collocation method to obtain the numerical solution with the use of Laguerre operational matrix.

The article is organized in following ways. Section 2 contains some important properties and definitions of fractional calculus. A brief overview of the conservation principle is given in Sect. 3. In Sect. 4, some outlines of Laguerre polynomials are given. In Sect. 5, an overview of Kronecker product and its properties are given. In Sects. 6 and 7, the Laguerre operational matrix for fractional order derivative in Caputo sense is given to solve our proposed model. The detail of convergence analysis of the proposed numerical scheme is given in Sect. 8. In Sect. 9, few examples to illustrate the accuracy and applicability of the proposed method are considered and comparisons between the numerical results obtained by our proposed method with the existing analytical solutions are shown through the graphs and tables. Numerical results and discussion of the considered two-dimensional nonlinear model are given in Sect. 10, which is followed by the outcomes of the overall research work given in the section Conclusion.

\section{Preliminaries}

In this section of the manuscript, the basic definitions and properties of variable order derivatives are given. The Riemann-Liouville's derivative has some drawbacks in the modeling the mathematical modeling for some real and physical phenomena. Fractional order derivative operator $D^{\eta}$ of the given order $\eta>0$ of a function $f(t)$ in the Caputo sense is given by [29, 41]

$$
\left(D^{\eta} f\right)(t)=\left\{\begin{array}{lc}
\frac{d^{r} f(t)}{d t^{r}}, & \text { if } \eta=r \in N, \\
\frac{1}{\Gamma(r-\eta)} \int_{0}^{t}(t-\rho)^{r-\eta-1} f^{(r)}(\rho) d \rho, & \text { if } r-1<\eta<r .
\end{array}\right.
$$

In the above expression $r$ is the integer number. Few properties of Caputo fractional derivatives are: 
$D^{\eta} C=0$,

where $C$ is an arbitrary constant.

$$
D^{\eta} t^{\varepsilon}= \begin{cases}0, & \text { if } \varepsilon \in N \cup\{0\}, \varepsilon<\lceil\eta\rceil, \\ \frac{\Gamma(\varepsilon+1)}{\Gamma(\varepsilon-\eta+1)} t^{\varepsilon-\eta}, & \text { if } \varepsilon \in N \cup\{0\}, \varepsilon \geq\lceil\eta\rceil \text { or } \varepsilon \notin N, \varepsilon>\lfloor\eta\rceil,\end{cases}
$$

where $\lceil\eta\rceil$ be the ceiling function and $\lfloor\eta\rfloor$ be the floor function.

\section{The general conservation principle}

In this section of the article, a fluid continuum in flowing state is considered. In general a multicomponent fluid has been considered in such a way that it follows the continuum approach and each component in itself is a continuum filling of the entire space, as in Fig. 1. Let $H_{a}$ be the initial amount of the the extensive fluid property (e.g., mass) is contained in a volume $V$ within a portion of space at time $t$ and enclosed by a surface $S$. Consider the position of the system at time $t+\triangle t$, in the time interval $\triangle t$, the system moves and gets deformed. At time $t$, the system is of surface area $S$ and volume $V$, whereas the system at time $t+\Delta t$ is of volume $V^{\prime}$ and surface $S^{\prime}$. These are composed of the regions: $V=V_{1}+V_{2}, V^{\prime}=V_{2}+V_{3}$. The region $V_{2}$ is common to $V$ and $V^{\prime}$.

In Lagrange point of view, the temporal rate of change $(D / D t) H_{a}$ of $H_{a}$ for the moving system is given by

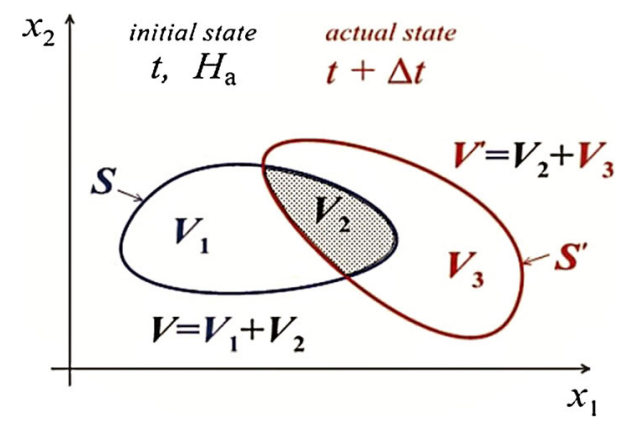

Fig. 1 Fluid continuum in the flowing process

$$
\begin{aligned}
& \left.\frac{D H_{a}}{D t}\right|_{\text {system }} \\
& \quad=\lim _{\triangle t \rightarrow \infty}\left\{\frac{\left[\left(H_{a}\right)_{2}+\left(H_{a}\right)_{3}\right]_{t+\triangle t}-\left[\left(H_{a}\right)_{1}+\left(H_{a}\right)_{2}\right]_{t}}{\triangle t}\right\},
\end{aligned}
$$

where

$$
\begin{gathered}
\left(H_{a}\right)_{i}=\int_{\left(V_{i}\right)} \rho_{a} \gamma_{a} d V=\int_{\left(V_{i}\right)} h_{a} d V, \\
i=1,2,3 ; H_{a} / \delta V=h_{a} .
\end{gathered}
$$

The general conservation principle of a property for the species ' $a$ ' is given by

$$
\partial h_{a} / \partial t+\operatorname{div}\left(h_{a} V_{H_{a}}\right)=I_{a}
$$

where $I_{a}$ is the temporal rate per unit volume and $V_{H_{a}}$ is the velocity field.

\subsection{Mass conservation of species}

Mass conservation of the fluid across a porous material involves the fundamental principle that total mass flux entered minus total mass flux out equals to the increase in mass amount stored by the medium. This means that the total mass of fluid is conserved. For a system of multicomponent fluid of a species ' $a$ ', substituting $h_{a}=\rho_{a}$ and $V_{H_{a}}=V_{a}$ in above equation, we get

$\partial \rho_{a} / \partial t+\operatorname{div}\left(\rho_{a} V_{a}\right)=I_{a}$,

where $I_{a}$ is the rate at which mass of the species ' $a$ ' produced per unit volume during the chemical reaction in the system. From the Eulerian point of view, above equation is the equation of continuity (or equation of mass conservation) [21].

If we assume the case of stationary fluid and $I_{a}=0$ then from above, we deduce the Fick's second law for diffusion for binary fluid as

$\partial \rho_{a} / \partial t=\operatorname{div}\left(D_{a b} \operatorname{grad} \rho_{a}\right)$,

where $D_{a b}$ is the diffusion coefficient.

\section{Laguerre polynomials and its some properties}

The involvement of Laguerre polynomial in several aspects of engineering and applied mathematics seeks the attention of many researchers and establishes it as a strong tool for finding the numerical solution of 
integer as well as fractional order partial differential equations (PDEs). The $l^{\text {th }}$ degree Laguerre polynomial is defined as [40]

$$
L_{l}(t)=\frac{1}{l !} e^{t} \partial_{t}^{l}\left(t^{l} e^{-t}\right), \quad l=0,1, \ldots ;
$$

The Laguerre polynomial of degree $l$ on the interval $I$ is simplified from above as

$$
L_{l}(t)=\sum_{k=0}^{l} \frac{l !(-1)^{k}}{(-k+l) !(k !)^{2}} x^{k}, \quad k=0,1, \ldots ;
$$

The following recurrence relations are satisfied by Laguerre polynomial

$$
\begin{aligned}
\partial_{t}\left(t e^{-t} \partial_{t} L_{l}(t)\right)+l e^{-t} L_{l}(t) & =0, \quad t \in I, \\
L_{l}(t) & =\partial_{t} L_{l}(t)-\partial_{t} L_{l+1}(t), \quad l \geq 0, \\
L_{l+1}(t) & =\frac{(2 l+1-t) L_{l}(t)-l L_{l-1}(t)}{l+1}, \\
& \forall l \geq 1 .
\end{aligned}
$$

The set of all Laguerre polynomials form an orthogonal system in $L_{r}^{2}(I)$ i.e.,

$$
\int_{I} L_{a}(t) L_{b}(t) r(t) d t=\delta_{l m}, \forall a, b \geq 0,
$$

where $r(t)=e^{-t}$ is weight function and $\delta_{l m}$ is the Kronecker delta function.

\section{Kronecker Product and its some properties}

In this section some basic and fundamental properties of the well known Kronecker product are defined $[9,19]$. Furthermore, we try to give some overview of the applications and properties of the Kronecker Product, which is an operation between two arbitrary size matrices represented by the symbol $\otimes$.

Definition The Kronecker product of the matrix $A$ of order $p \times q$ and $B$ of order $m \times n$ is again $p m \times q n$ order block matrix, which is denoted by $A \otimes B$ and is defined by

$$
A \otimes B=\left[\begin{array}{cccc}
a_{11} B & a_{12} B & \ldots & a_{1 q} B \\
a_{21} B & a_{22} B & \ldots & a_{21} B \\
\vdots & \vdots & \vdots & \vdots \\
a_{p 1} B & a_{p 2} B & \ldots & a_{p q} B
\end{array}\right] .
$$

Moreover, If $A$ and $B$ represent some linear transformations on vector spaces i.e., $W_{1} \rightarrow V_{1}$ and $W_{2} \rightarrow$ $V_{2}$ respectively, then $A \otimes B$ represents the tensor product of these two maps, $W_{1} \otimes W_{2} \rightarrow V_{1} \otimes V_{2}$.

\subsection{Basic properties}

Since Kronecker product is a special case of tensor product, therefore it can satisfy the property like associativity and bi-linearity. Also, it has a lot of interesting and effective properties, few are stated below:

$$
\begin{aligned}
& I \otimes(J+K)=I \otimes J+I \otimes K, \\
& (I+J) \otimes K=I \otimes K+J \otimes K, \\
& (k I \otimes J)=(I \otimes k J)=k(I \otimes J), \\
& (I \otimes J) \otimes K=I \otimes(J \otimes K),
\end{aligned}
$$

where $I, J$ and $K$ are arbitrary matrices and $k$ is a scalar.

\section{Laguerre operational matrix for fractional order differentiation}

The collection of Laguerre polynomials form a basis set for $L_{r}^{2}(I)$. So any function $\zeta(t) \in L_{r}^{2}(I)$ can be expanded as

$$
\zeta(t)=\sum_{g=0}^{\infty} b_{g} L_{g}(t), m=0,1,2, \ldots
$$

where the unknown constants $b_{g}$ can be calculated as $b_{g}=\int_{0}^{\infty} \zeta(t) L_{b}(t) r(t) d t$.

In the terms of first $(r+1)$ Laguerre polynomials the above approximation is reduced to

$$
\zeta_{r}(t)=\sum_{g=0}^{r} b_{g} L_{g}(t)=B^{T} \cdot \sigma(t),
$$

where $B^{T}=\left[b_{0}, b_{1}, \ldots, b_{r}\right]$ is the matrix of unknown constants and $\sigma(t)=\left[L_{0}(t), L_{1}(t), \ldots, L_{r}(t)\right]^{T}$ is the Laguerre vector. Again a function of two variables 
$\zeta(x, t) \in L^{2}[0,1]$ can be expressed in terms of Laguerre polynomials as

$\zeta(x, t) \simeq \zeta_{r}(x, t)=\sum_{g=0}^{r} \sum_{h=0}^{r} b_{g, h} L_{g}(x) L_{h}(t)=\sigma(x)^{T} . B . \sigma(t)$,

where the unknown constant matrix $B=\left[b_{g, h}\right]$ is known as Laguerre coefficient.

In similar manner, we can approximate an arbitrary two-dimensional function $\zeta(x, y, t) \in L^{2}[0,1]$ in terms of Laguerre polynomial as

$$
\begin{aligned}
\zeta(x, y, t) \simeq \zeta_{r}(x, y, t) & =\sum_{g=0}^{r} \sum_{h=0}^{r} \sum_{i=0}^{r} b_{g, h, i} \Omega_{g}(x) L_{h}(y) L_{i}(t) \\
& =\sigma(t)^{T} \cdot B \cdot \sigma(x) \otimes \sigma(y),
\end{aligned}
$$

where the unknown constant matrix $B=\left[b_{g, h, i}\right]$ is the Laguerre coefficient. These coefficients can be determine by using the initial and boundary conditions. In order to derive the novel operational matrix we have provided the following lemma.

Lemma 1 Consider the fractional derivative operator $D^{\mu}$ of fractional order $\mu$ in Caputo sense. Let $\sigma(t)$ be the Laguerre vector defined as in equation (30), then we have following results:

${ }_{0}^{C} D_{t}^{\mu} L_{r}(t)=0, \quad r=0,1, \ldots,\lceil\mu\rceil-1, \quad 0<\mu \leq 1$.

Proof This result can be easily proved by using the basic properties of fractional Caputo derivative given in the equation (13).

We are going to derive the operational matrix of orthogonal Laguerre polynomials [40]. On solving the equations (28)-(29) and equation (20), we get the following expression as

$$
\begin{aligned}
{ }_{0}^{C} D_{t}^{\mu} L_{l}(t)= & \sum_{g=0}^{l} \\
& \frac{(-1)^{g} ! ! D^{\mu} t^{g}}{(l-g) !(g !)^{2}}=\sum_{g=\lceil\mu\rceil}^{l} \frac{(-1)^{g} l ! t^{g-\mu}}{(l-g) ! \Gamma(g-\mu+1) g !}, \quad l=\lceil\mu\rceil, \ldots, r .
\end{aligned}
$$

As set of Laguerre polynomials is a basis so we can write the term $t^{g-\mu}$ as

$$
t^{g-\mu}=\sum_{h=0}^{r} b_{h} L_{h}(t),
$$

where the constant coefficient $b_{h}$ is given by $b_{h}=\sum_{a=0}^{h} \frac{(-1)^{a} h ! \Gamma(g-\mu+a+1)}{(h-a) !(a !)^{2}}$.

Using the equations (35)-(36) into the equation (34), we have

${ }_{0}^{C} D_{t}^{\mu} L_{l}(x)=\sum_{h=0}^{r} P_{\mu}(l, h) L_{h}(t), \quad l=\lceil\mu\rceil, \ldots, r ;$

In the above equation the term $P_{\mu}(l, h)$ is given by the following equation

$$
P_{\mu}(l, h)=\sum_{g=\lceil\mu\rceil}^{l} \sum_{a=0}^{h} \frac{(-1)^{g+a} l ! h ! \Gamma(g-v+a+1)}{(l-g) ! \Gamma(g-\mu+1) g !(h-a) !(a !)^{2}} .
$$

The vector form of the equation (37) is written as

$$
\begin{aligned}
& { }_{0}^{C} D_{t}^{\mu} L_{l}(t)=\left[P_{\mu}(l, 0), P_{\mu}(l, 1), \ldots, P_{\mu}(l, r)\right] \sigma(t), \\
& \quad l=\lceil\mu\rceil, \ldots, r .
\end{aligned}
$$

In the view of lemma 1 , we have

$$
{ }_{0}^{C} D_{t}^{\mu} L_{l}(t)=[0,0, \ldots, 0] \sigma(x), \quad l=0,1, \ldots,\lceil\mu\rceil-1 .
$$

Using the equations (39)-(40), we can write the equation (40) in matrix form as

$$
{ }_{0}^{C} D_{t}^{\mu} \sigma(t)=H^{(\mu)} \sigma(t),
$$

where $H^{\mu}$ is an operational matrix with fractional derivatives of $r+1 \times r+1$ order and is given as

$$
H^{(\mu)}=\left[\begin{array}{ccccc}
0 & 0 & 0 & \ldots & 0 \\
\vdots & \vdots & \vdots & \ldots & \vdots \\
0 & 0 & 0 & \ldots & 0 \\
P_{\mu}(\lceil\mu\rceil, 0) & S_{\mu}(\lceil\mu\rceil, 1) & P_{\mu}(\lceil\mu\rceil, 2) & \ldots & P_{\mu}(\lceil\mu\rceil, r) \\
\vdots & \vdots & \vdots & \ldots & \vdots \\
P_{\mu}(i, 0) & P_{\mu}(i, 1) & P_{\mu}(i, 2) & \ldots & P_{\mu}(i, r) \\
\vdots & \vdots & \vdots & \ldots & \vdots \\
P_{\mu}(r, 0) & P_{\mu}(r, 1) & P_{\mu}(r, 2) & \ldots & P_{\mu}(r, r)
\end{array}\right],
$$

\section{Implementation of Laguerre operational matrix}

Here we are going to apply the fractional operational matrix on concerned two-dimensional mathematical model using Laguerre collocation method. 
Now, in view of the equation (32), we shall approximate the solute concentration $u(x, y, t)$ in form of Laguerre polynomials in the following manner as

$u(x, y, t)=\sum_{f=0}^{r} \sum_{g=0}^{r} \sum_{h=0}^{r} c_{f g h} L_{f}(x) L_{g}(y) L_{h}(t)$,

where $c_{g h i}$ is the Laguerre coefficients, where $g=$ $1,2,3, \ldots ; h=1,2,3, \ldots ; i=1,2,3, \ldots ;$ The equation (43) can be represented in vector form as

$u(x, y, t)=\sigma(t)^{T} \cdot B \cdot \sigma(x) \otimes \sigma(y)$,

where $B=\left[c_{f g h}\right]$ is a matrix of order $(r+1) \times(r+$ $1)^{2}$ of constant Laguerre coefficients and $\sigma(t)=$ $\left[L_{0}(t), L_{1}(t) \ldots L_{r}(t)\right]^{T}$ is a column vector. Applying the fractional derivative of order $\alpha$ with respect to time variable variable $t$ on equation (44) and using the equation (41), we get

$\frac{\partial^{\alpha} u}{\partial t^{\alpha}}=H^{\alpha} u(x, y, t)=\left[H^{\beta} \cdot \sigma^{T}(t)\right] \cdot B \cdot \sigma(x) \otimes \sigma(y)$.

Similarly,

$$
\frac{\partial u}{\partial x}=H u(x, y, t)=\sigma^{T}(t) \cdot B \cdot\left(H^{1} \times I\right) \cdot \sigma(x) \otimes \sigma(y),
$$

$\frac{\partial u}{\partial y}=H u(x, y, t)=\sigma^{T}(t) \cdot B \cdot\left(I \times H^{1}\right) \cdot \sigma(x) \otimes \sigma(y)$.

In above equations $I$ be the $(r+1) \times(r+1)$ identity matrix. Now from prescribed initial and boundary conditions (5)-(9) with the aid of equation (44), we get

$$
\begin{aligned}
& \sigma^{T}(0) \cdot B \cdot \sigma(x) \otimes \sigma(y)=0, \\
& \sigma^{T}(t) \cdot B \cdot \sigma(0) \otimes \sigma(y)=c_{0}, \\
& \sigma^{T}(t) \cdot B \cdot\left(H^{1} \times I\right) \cdot \sigma(1) \otimes \sigma(y)=0, \\
& \sigma^{T}(t) \cdot B \cdot\left(I \times H^{1}\right) \cdot \sigma(x) \otimes \sigma(0)=0, \\
& \sigma^{T}(t) \cdot B \cdot\left(I \times H^{1}\right) \cdot \sigma(x) \otimes \sigma(1)=0 .
\end{aligned}
$$

Now, we collocate Eq. (4) with the help of equations (48-52) at points $x_{b}=\frac{b}{r}$ for $b=0,1,2, \ldots, r, y_{r}=\frac{b}{r}$ for $b=0,1,2, \ldots, r$ and $t_{r}=\frac{b}{r}$ for $b=0,1,2, \ldots, r$.
Using these collocation points and fractional operational matrix, a nonlinear system of algebraic equations are found. On simplifying this system we can calculate the Laguerre coefficients matrix $B$ which can further be used in Eq. (44) to find the approximate numerical solution.

\section{Error bound of the approximation}

In this section the upper bound of the error of proposed numerical approximation is estimated.

Theorem 1 For the generalized Laguerre polynomial $L_{l}^{(\theta)}(x)$, the global uniform bounds can be given by

$$
\left|L_{l}^{(\theta)}(x)\right| \leq\left\{\begin{array}{cc}
\frac{(\theta+1)_{l}}{l !} e \frac{x}{2}, & \text { if } \theta \geq 0, x \geq 0, l=0,1,2, \ldots ; \\
\left(2-\frac{(\theta+1)}{l !}\right) e \frac{x}{2}, & \text { if }-1<\theta \leq 0, x \geq 0, l=0,1,2, \ldots
\end{array}\right.
$$

where

$$
(b)_{l}=b(b+1)(b+2) \ldots(b+l-1), l=1,2,3, \ldots ;
$$

Proof M. Abramowitz and I. A. Stegun have proved the global uniform bounds in [2]. The estimates are also been discussed in [28].

Remark The global uniform bound (53) for the usual Laguerre polynomial is given as

$$
\left|L_{r}(x)\right| \leq \frac{1}{r !} e^{\frac{x}{2}}, \quad x \geq 0, \quad x=0,1,2, \ldots
$$

Theorem 2 Suppose $u(x, y, t)$ be the sufficiently smooth function on the region $A$ and $\left(\frac{\partial^{\alpha} u}{\partial t^{\alpha}}\right)_{r}$ be the approximation of $\left(\frac{\partial^{\alpha} u}{\partial t^{\alpha}}\right)$. Then the approximated error $\left(\frac{\partial^{\alpha} u}{\partial t^{\alpha}}\right)$ by $\left(\frac{\partial^{\alpha} u}{\partial t^{\alpha}}\right)_{r}$ is bounded by

$$
\begin{aligned}
& |E(r)| \leq \sum_{f=0}^{r} \sum_{g=0}^{r} \sum_{h=0}^{r} \frac{c_{f g h}}{f ! \cdot g ! . m !} \cdot \chi_{m h r} \cdot e^{\frac{x+y+t}{2}}, \\
& \qquad x, y, t \geq 0, \quad f, g, m=0,1,2, \ldots ; \\
& \text { where } \chi_{m b r}=\sum_{m=0}^{r} S_{\alpha}(b, m) .
\end{aligned}
$$

Proof In view of equation (43), we have 


$$
u(x, y, t)=\sum_{f=0}^{r} \sum_{g=0}^{r} \sum_{h=0}^{r} c_{f g h} L_{f}(x) L_{g}(y) L_{h}(t),
$$

truncating it upto $r+1$ terms, we have

$$
u_{r}(x, y, t)=\sum_{f=0}^{r} \sum_{g=0}^{r} \sum_{h=0}^{r} c_{f g h} L_{f}(x) L_{g}(y) L_{h}(t) .
$$

We can write $\alpha$ order partial derivative of $u(x, y, t)$ and $u_{r}(x, y, t)$ w.r.to $t$ as

$$
\begin{aligned}
& \frac{\partial^{\alpha} u}{\partial t^{\alpha}}=\sum_{f=0}^{r} \sum_{g=0}^{r} \sum_{h=0}^{r} c_{f g h} L_{f}(x) L_{g}(y) \frac{\partial^{\alpha} L_{h}(t)}{\partial t^{\alpha}}, \\
& \left(\frac{\partial^{\alpha} u}{\partial t^{\alpha}}\right)_{r}=\sum_{f=0}^{r} \sum_{g=0}^{r} \sum_{h=0}^{r} c_{f g h} L_{f}(x) L_{g}(y) \frac{\partial^{\alpha} L_{h}(t)}{\partial t^{\alpha}} .
\end{aligned}
$$

From the above equations, we can write

$$
\begin{aligned}
E(r) & =\frac{\partial^{\alpha} u}{\partial t^{\alpha}}-\left(\frac{\partial^{\alpha} u}{\partial t^{\alpha}}\right)_{r} \\
& =\sum_{f=0}^{r} \sum_{g=0}^{r} \sum_{h=0}^{r} c_{f g h} L_{f}(x) L_{g}(y) \frac{\partial^{\alpha} L_{h}(t)}{\partial t^{\alpha}} .
\end{aligned}
$$

Using (37), the above equation reduces to

$$
|E(r)|=\left|\sum_{f=0}^{r} \sum_{g=0}^{r} \sum_{h=0}^{r} c_{f g h} L_{f}(x) L_{g}(y)\left(\sum_{m=0}^{r} S_{\alpha}(b, m) L_{m}(t)\right)\right|
$$

or,

$$
\begin{aligned}
|E(r)|= & \sum_{f=0}^{r} \sum_{g=0}^{r} \sum_{h=0}^{r} c_{f g h} L_{g}(y) \\
& \cdot \chi_{m h r}\left|L_{f}(x)\right| \cdot\left|L_{g}(y)\right| \cdot\left|L_{m}(t)\right| .
\end{aligned}
$$

Applying equation (54), we get

$$
\begin{gathered}
|E(r)| \leq \sum_{f=0}^{r} \sum_{g=0}^{r} \sum_{h=0}^{r} c_{f g h} . \\
\chi_{m h r} \cdot \frac{1}{m !} e^{\frac{t}{2}} \cdot \frac{1}{f !} e^{\frac{x}{2}} \cdot \frac{1}{g !} e^{\frac{y}{2}} .
\end{gathered}
$$

Using the formulae S1 and S2 given in [31] and subtracting the truncated series from the infinite series, bounding each term in the difference, and summing the bounds complete the proof of the theorem.

$$
\begin{aligned}
& |E(r)| \leq \sum_{f=0}^{r} \sum_{g=0}^{r} \sum_{h=0}^{r} \frac{c_{f g h}}{f ! . g ! . m !} \\
& \cdot \chi_{m h r} . e^{\frac{x+y+t}{2}}, \quad x, y, t \geq 0, \quad f, g, m=0,1,2, \cdots
\end{aligned}
$$

\section{Numerical simulations and error analysis}

Laguerre fractional operational matrix has been applied on some existing fractional order problems to find their approximate numerical solutions. The obtained numerical results have been compared with the existing analytical solutions through finding the absolute error for approximation to illustrate the capability and validity of the proposed numerical scheme. All the numerical calculations are carried out by using the software MATHEMATICA 11.3.

The absolute error is defined by [16] as

$$
E_{a b s}(x, y, t)=\left|u_{\text {exact }}-u_{\text {num }}(x, y, t)\right| \text {. }
$$

Maximum error for the problem in $x$ for fixed $y$ and $t$ is defined as

$$
\operatorname{Max} E_{a b s}(x, 0.5,0.5)=\max _{0 \leq x \leq 1}\left|E_{a b s}(x, 0.5,0.5)\right| .
$$

The absolute for the concerned test examples with a fix value of $t$ and $x$ is given by

$$
\operatorname{Max} E_{a b s}(0.5, y, 0.5)=\max _{0 \leq y \leq 1}\left|E_{a b s}(0.5, y, 0.5)\right| .
$$

The order of convergence for two successive approximation $N_{1}$ and $N_{2}$ is also calculated to validate the higher order accuracy of the numerical scheme which is given as

$$
\text { Order }=\frac{\log \left(\frac{\operatorname{Max} E_{a b s}\left(N_{1}\right)}{\operatorname{Max} E_{a b s}\left(N_{2}\right)}\right)}{\log \left(\frac{N_{2}}{N_{1}}\right)},
$$

where $\operatorname{Max} E_{a b s}(N)$ is the maximum absolute error during the approximation of degree $N$. 
Example 1 Let us consider a two-dimensional nonlinear non-homogeneous fractional order advectiondiffusion equation as

$$
\frac{\partial^{\alpha} u}{\partial t^{\alpha}}=\frac{\partial^{2} u}{\partial x^{2}}+\frac{\partial^{2} u}{\partial y^{2}}+\frac{\partial u}{\partial x}+\frac{\partial u}{\partial y}+\frac{u^{2}}{3}+f(x, y, t),
$$

with $f(x, y, t)=-x \sqrt{t}-y \sqrt{t}-\frac{x^{2} y^{2} t}{3}-\frac{0.682734 x y}{t^{0.3}}$ and boundary conditions

$$
\begin{aligned}
& u(x, y, 0)=0, \\
& u(0, y, t)=0, \\
& u(x, 0, t)=0, \\
& u(1, y, t)=y \sqrt{t}, \\
& u(x, 1, t)=x \sqrt{t} .
\end{aligned}
$$

For the above example, the exact solution is $u(x, y, t)=x y \sqrt{t}$ for the fractional order parameters $\alpha=0.8$. The absolute errors between the results obtained by our proposed numerical scheme and the exact solutions are exhibited through Tables 1 and 2 for $y=0.5,0 \leq x \leq 1$ and $x=0.5,0 \leq y \leq 1$ respectively for the order of approximation $N=5,7,9$ at $t=1$. The tables clearly confirm that the order of convergence for our proposed method increases as the degree of Laguerre polynomials in $x$ and $y$ increases. Again absolute error decreases with the increase of the order of approximation of the polynomial $N$, which clearly shows the effectiveness of our proposed numerical method. Again the data of CPU time shows that it takes minimum time to obtain accurate result showing that the method is computationally effective to solve the two-dimensional fractional order PDEs.

The absolute error is graphycally shown through Fig. 2 for various values of $y$ and $t$ for a fixed value of $x=0.5$. Figure 2 shows that the numerical solution is approximately accurate to the exact solution even for small value $N=5$, the better convergence can be achieved increasing $N$. Thus comparing the numerical solution with the exact solution, we can say that the proposed method is very much efficient.

Example 2 We consider time-fractional non-linear Fisher's equation as

$$
\frac{\partial^{\alpha} u}{\partial t^{\alpha}}=\frac{1}{2} \nabla^{2} u(x, y, t)+u^{2}(1-u)+f(x, y, t),
$$

where $\quad f(x, y, t)=e^{x y}\left(1.91116 t^{1.1}+e^{x y} t^{4}(-1+\right.$ $\left.\left.e^{x y} t^{2}\right)-0.5 t^{2}\left(x^{2}+t^{2}\right)\right)$ with the following initial and boundary conditions as

$$
\begin{aligned}
& u(x, y, 0)=0, \\
& u(0, y, t)=t^{2}, \\
& u(x, 0, t)=t^{2}, \\
& u(1, y, t)=e^{y} t^{2}, \\
& u(x, 1, t)=e^{x} t^{2} .
\end{aligned}
$$

The exact solution of this time-fractional Fisher's equation is $u(x, y, t)=e^{x y} t^{2}$ for the fractional order time parameter $\alpha=0.9$. The absolute errors, order of convergence and the CPU time required for the PDE with our proposed method are given in Tables 3 and 4 for $y=0.5,0 \leq x \leq 1$ and $x=0.5,0 \leq y \leq 1$ respectively for the order of approximation $N=3,5,7$ at $t=1$. The tables clearly exhibit that our proposed scheme is computationally effective even for less CPU time.

The absolute error between the numerical solution and the exact solution is depicted through Fig. 3 at $t=1$ for various values of $x$ and $y$.

Figure 3 shows that the numerical solution is approximately accurate to the exact solution even for $N=5$. The better convergence can be achieved with increase in $N$.

Example 3 Consider following two-dimensional non-linear non-homogeneous fractional order advection-reaction-diffusion equation as

$$
\frac{\partial^{\alpha} u}{\partial t^{\alpha}}=\frac{\partial^{2} u}{\partial x^{2}}+\frac{\partial^{2} u}{\partial y^{2}}+\frac{\partial u}{\partial x}+\frac{\partial u}{\partial y}+u(1-u)+f(x, y, t),
$$

where $\quad f(x, y, t)=1.91116 t^{1.1} x^{2} y^{2}+t^{4} x^{4} y^{4}+$ $t^{2}\left(-2 x y^{2}-2 y^{2}+x^{2}\left(-2-y^{2}\right)\right)-2 x^{2} y t^{2}$ with the following initial and boundary conditions 
Table 1 Efficiency of the numerical method for Example 1 at $y=t=0.5$

\begin{tabular}{lllll}
\hline $\mathrm{y}$ & $\mathrm{N}$ & Maximal absolute error $0 \leq x \leq 1$ & Order of convergence & CPU TIME (second) \\
\hline 0.5 & 5 & 0.00063 & - & 7.22 \\
& 7 & 0.000098 & 5.53 & 8.48 \\
& 9 & 0.000008 & 9.96 & 12.39 \\
\hline
\end{tabular}

Table 2 Efficiency of the numerical method for Example 1 at $x=t=0.5$

\begin{tabular}{lllll}
\hline $\mathrm{x}$ & $\mathrm{N}$ & Maximal absolute error $0 \leq y \leq 1$ & Order of convergence & CPU Time (s) \\
\hline 0.5 & 5 & 0.00071 & - & 7.21 \\
& 7 & 0.000084 & 6.27 & 8.48 \\
& 9 & 0.0000071 & 9.92 & 12.39 \\
\hline
\end{tabular}

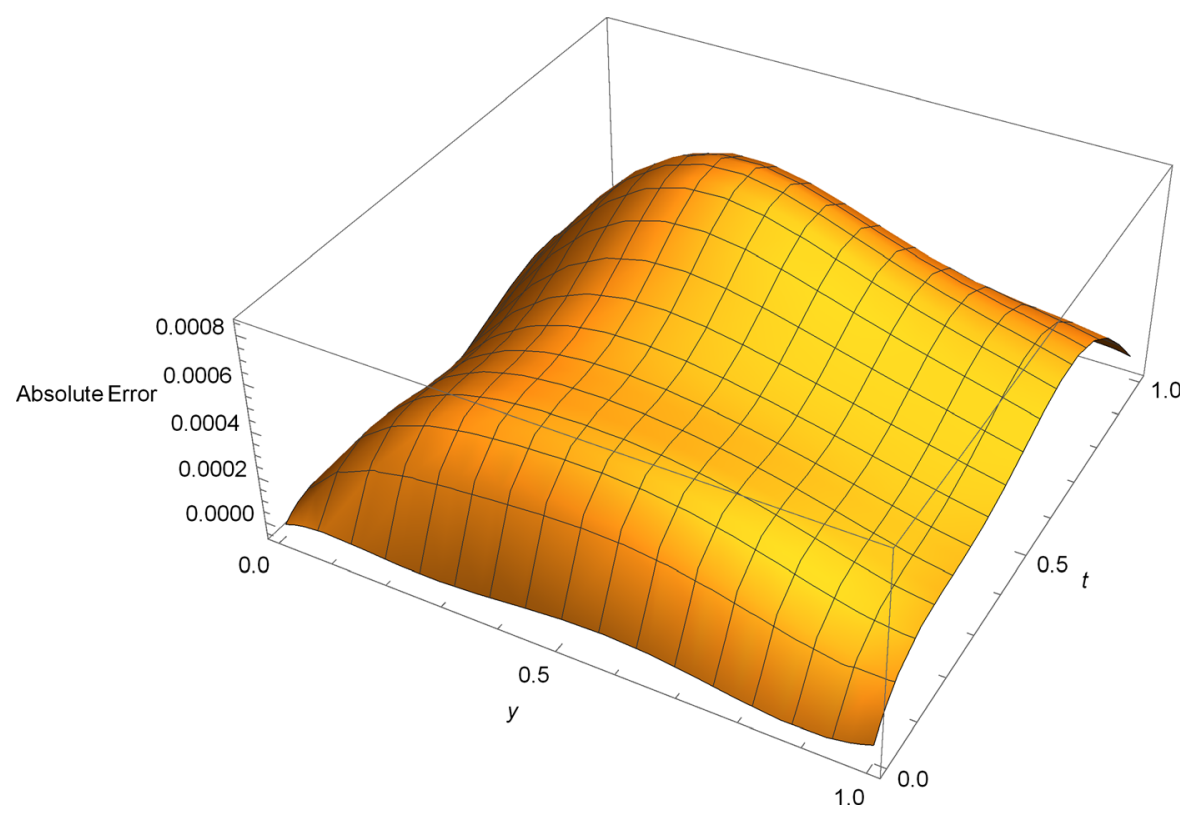

Fig. 2 Plot of the absolute error between the exact and numerical solutions vs. $y$ and $t$ at $x=0.5$

Table 3 Efficiency of the numerical method for Example 2 at $y=t=0.5$

\begin{tabular}{lllll}
\hline $\mathrm{y}$ & $\mathrm{N}$ & Maximal absolute error $0 \leq x \leq 1$ & Order of convergence & CPU TIME (s) \\
\hline 0.5 & 5 & 0.00026 & - & 7.12 \\
& 7 & 0.000041 & 5.48 & 9.36 \\
& 0.0000067 & 7.20 & 10.55 \\
\hline
\end{tabular}


Table 4 Efficiency of the numerical method for Example 2 at $x=t=0.5$

\begin{tabular}{lllll}
\hline $\mathrm{x}$ & $\mathrm{N}$ & Maximal absolute error $0 \leq y \leq 1$ & Order of convergence & CPU Time (s) \\
\hline 0.5 & 5 & 0.0003 & - & 7.10 \\
& 7 & 0.000039 & 6.06 & 9.41 \\
& 9 & 0.0000064 & 7.19 & 10.48 \\
\hline
\end{tabular}

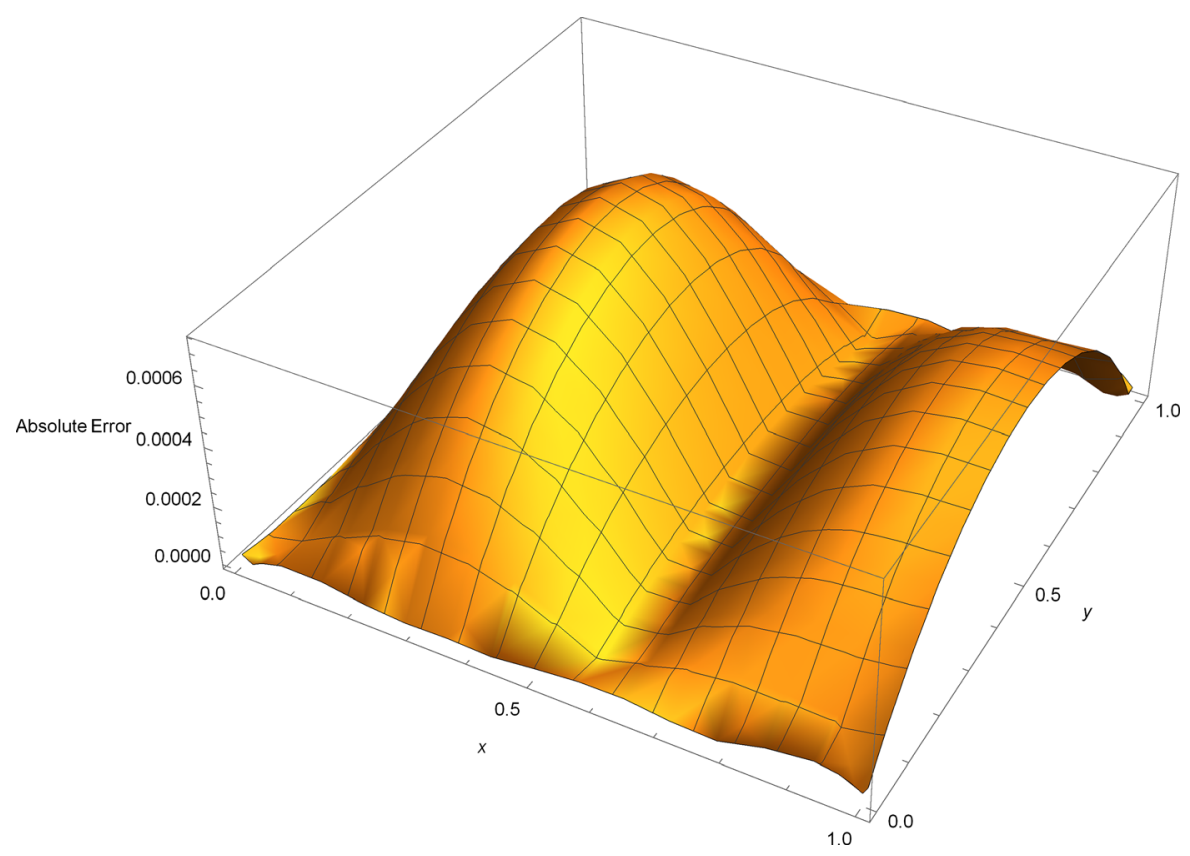

Fig. 3 Plot of the absolute error between the exact and numerical solutions vs. $x$ and $y$ at $t=1$.

$$
\begin{gathered}
u(x, y, 0)=0, \\
u(0, y, t)=0, \\
u(x, 0, t)=0, \\
u(1, y, t)=y^{2} t^{2}, \\
u(x, 1, t)=x^{2} t^{2} .
\end{gathered}
$$

The exact solution for this concerned example is $u(x, y, t)=x^{2} y^{2} t^{2}$ for fractional order time parameter $\alpha=0.9$. The absolute errors, order of convergence and the CPU time required for the PDE with our proposed method are given in Tables 5 and 6 for $y=$ $0.5,0 \leq x \leq 1$ and $x=0.5,0 \leq y \leq 1$ respectively for the order of approximation $N=5,7,9$ at $t=1$. It is clear from the tables that our proposed scheme is computationally effective even for less CPU time.
The absolute error between the numerical solution and the exact solution is depicted through Fig. 4 at $t=1$ for various values of $x$ and $y$.

Figure 4 shows that the numerical solution is approximately accurate to the exact solution even for $N=5$. The better convergence can be achieved with increase in $N$.

\section{Results and discussion for proposed model}

After being a justification of the accuracy and effectiveness of the method, the authors have been motivated to apply their proposed numerical scheme to solve the concerned two-dimensional nonlinear time fractional order reaction-diffusion model (4) under the prescribed initial and boundary conditions (5)-(9). The variations of the solute 
Table 5 Efficiency of the numerical method for Example 3 at $y=t=0.5$

\begin{tabular}{lllll}
\hline $\mathrm{y}$ & $\mathrm{N}$ & Maximal absolute error $0 \leq x \leq 1$ & Order of convergence & CPU TIME (s) \\
\hline 0.5 & 5 & 0.00033 & - & 7.47 \\
& 7 & 0.000028 & 7.33 & 10.54 \\
& 9 & 0.0000022 & 10.12 & 12.35 \\
\hline
\end{tabular}

Table 6 Efficiency of the numerical method for Example 3 at $x=t=0.5$

\begin{tabular}{lllll}
\hline $\mathrm{x}$ & $\mathrm{N}$ & Maximal absolute error $0 \leq y \leq 1$ & Order of convergence & CPU Time (s) \\
\hline 0.5 & 5 & 0.00038 & - & 7.47 \\
& 7 & 0.000032 & 7.35 & 10.53 \\
& 9 & 0.0000026 & 9.98 & 12.35 \\
\hline
\end{tabular}

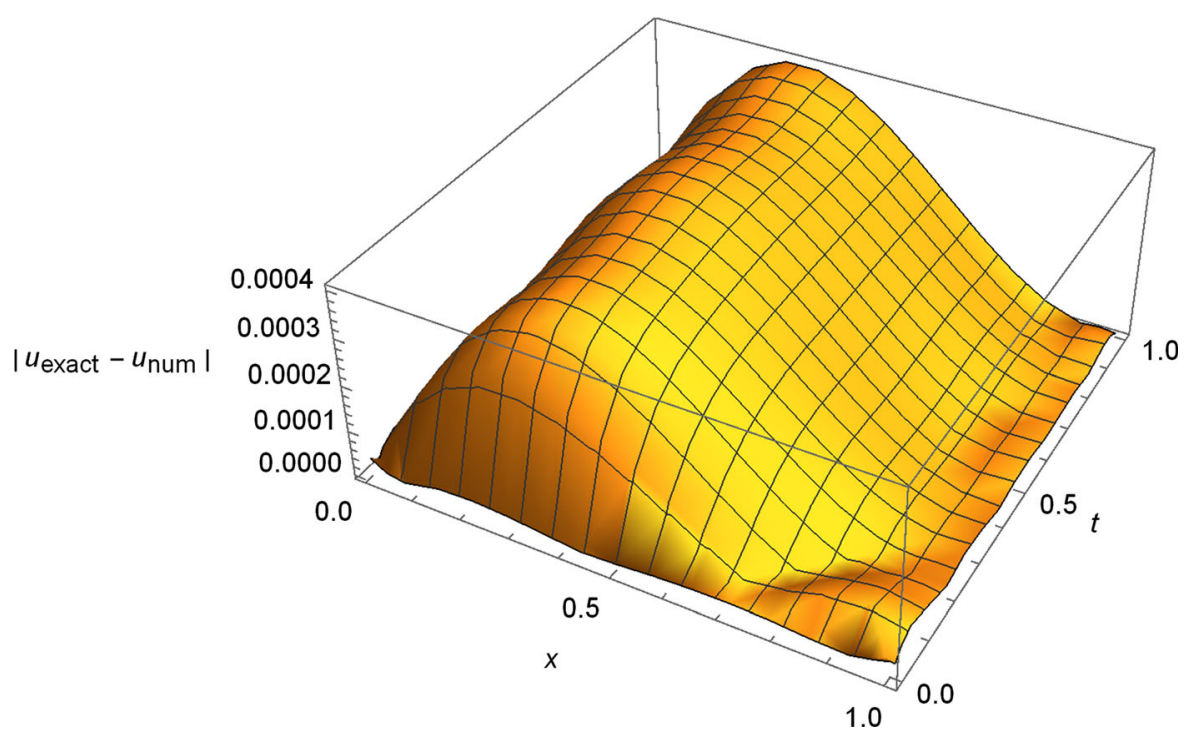

Fig. 4 Plot of the absolute error between the exact and numerical solutions vs. $x$ and $t$ at $y=0.5$.

concentration $u(x, y, t)$ vs. the column length $x$ and $y$ at $t=0.5$ for various values of the fractional order time parameter $\alpha$ for conservative case $(k=0)$ and nonconservative case $(k \neq 0)$ are calculated numerically and are displayed graphically through Figs. 5, 6, and 7.

The effects of reaction term on the solution profile for various values of the fractional order time derivative are found for $k=-1$ and $k=0$, which are shown through Figs. 5 and 6 respectively. It is seen that the overshoots of sub-diffusion are decreased as the system advances towards fractional order from standard order. It is also seen from the figures that dampings are found due the presence of sink term $(k=-1)$. Figure 7 reveals that it consumes less time to stabilize the probability density function $u(x, y, t)$ at $t=0.5$ for the case $\alpha=0.7$ due to the effect of sink term $k=-1$ as compared to the case of $k=0$ and also the presence of source term $k=1$, which clearly shows that physical importance of the presence of sink term in the system to enhance the stability region. 


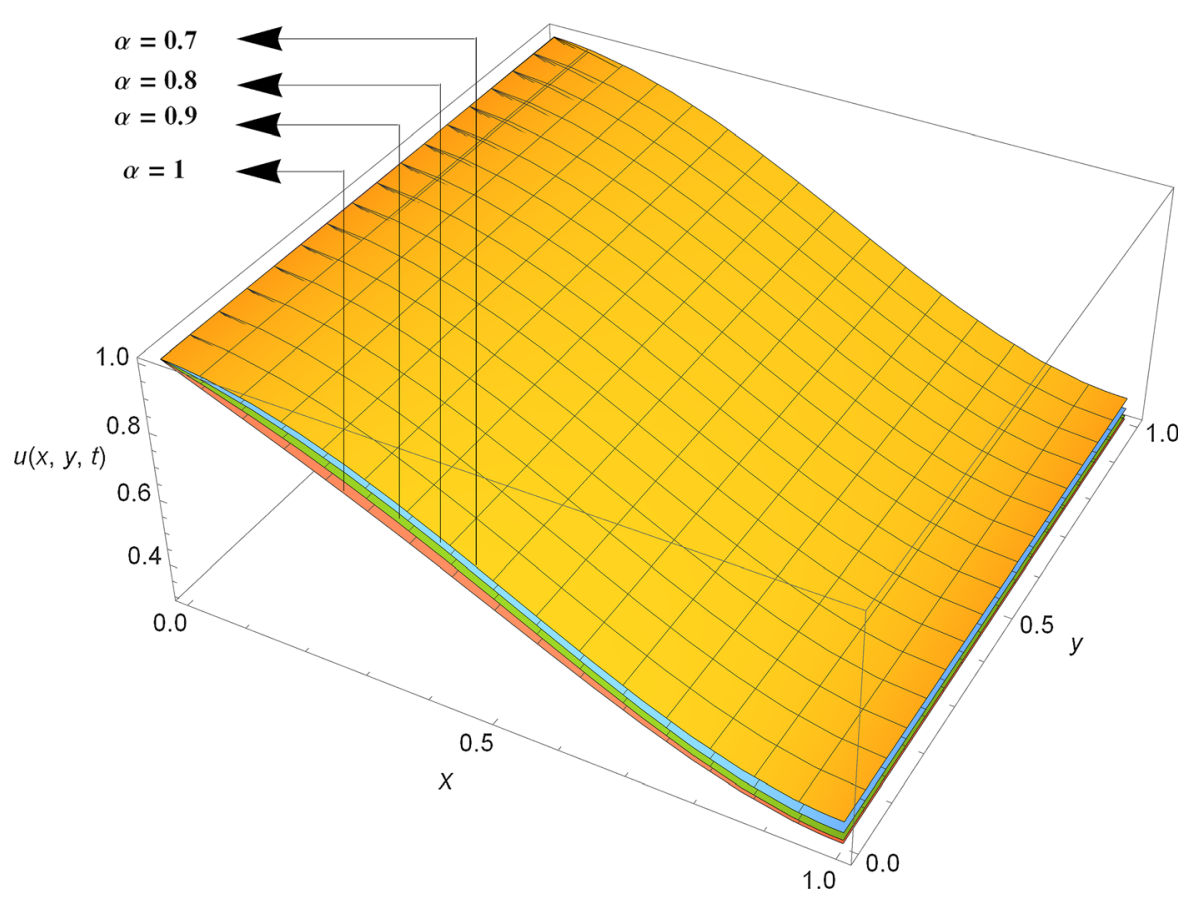

Fig. 5 Plots of the field variable $u(x, y, t)$ vs. $x$ and $y$ at $t=0.5$ for $k=-1$ for different values of $\alpha$

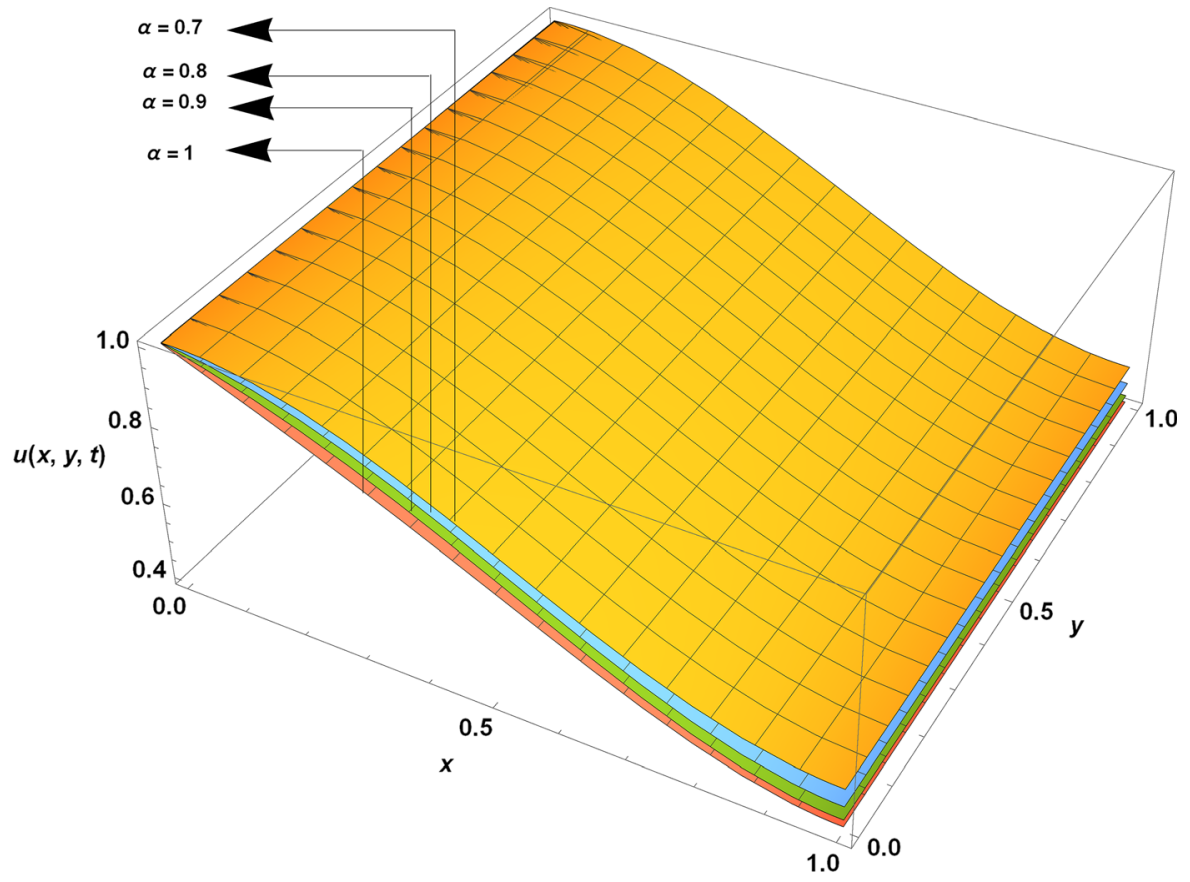

Fig. 6 Plots of the field variable $u(x, y, t)$ vs. $x$ and $y$ at $t=0.5$ for $k=0$ for different values of $\alpha$ 


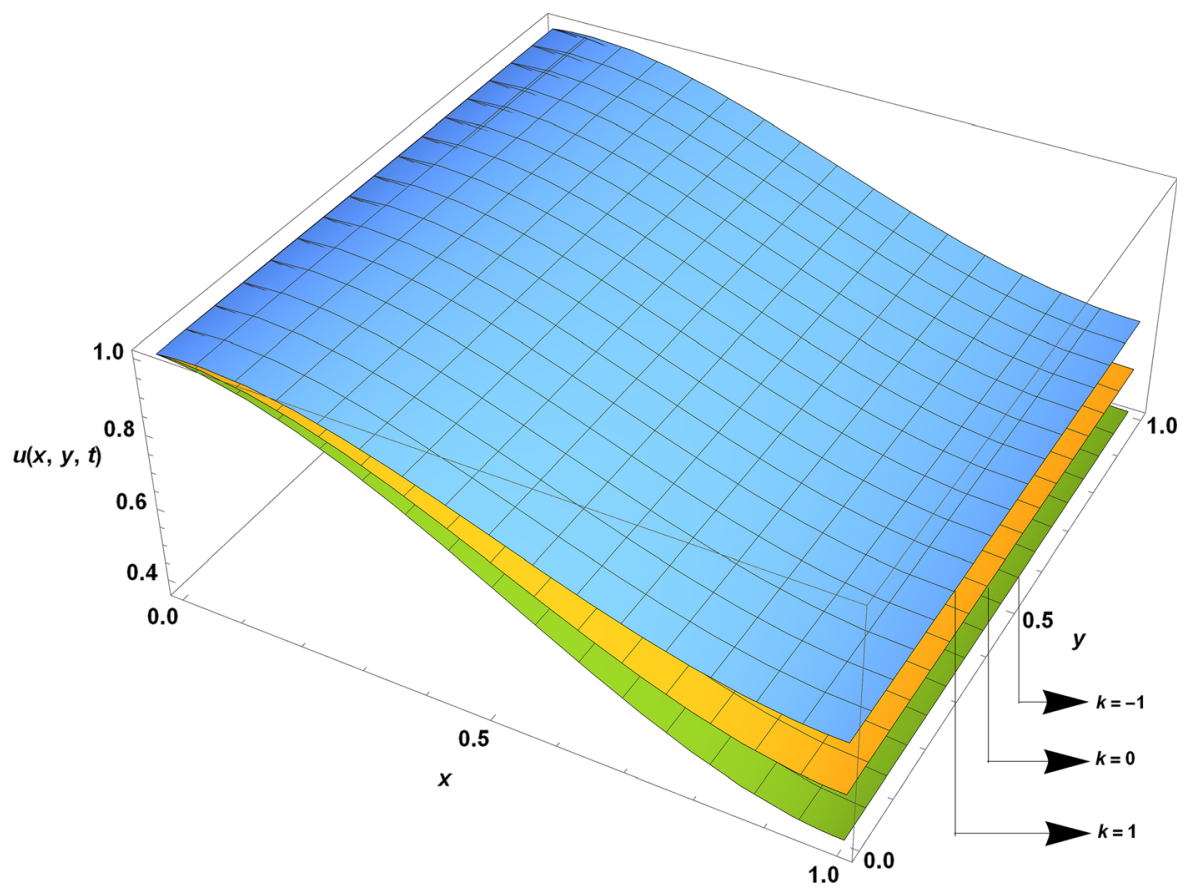

Fig. 7 Plots of field variable $u(x, y, t)$ vs. $x$ and $y$ at $t=0.5$ for $\alpha=0.7$ for different values of $k$

\section{Conclusions}

Four objectives have been accomplished through the present scientific contribution. First one is finding the solute concentration $u(x, y, t)$ for two-dimensional non-linear fractional order reaction-diffusion equation employing the powerful and efficient technique Laguerre operational matrix. Second one is the pictorial presentations of the nature of overshoots in the form of sub-diffusion due to presence of reaction terms for different particular cases. The third one is the graphical presentations of the damping nature of the solute concentration when the system advances towards fractional order from standard order in the presence of sink term. The most important part of the present study is the exhibition of the order of convergence during validation of the proposed method while applying on the existing problems having analytical solutions. The FDE model has already been applied to describe heat transfer in ceramic polycrystalline composite materials made of two phases $[49,51,38]$, in metal matrix composites $[42,50,10,54,55]$ or in ceramic porous materials $[52,53]$. The authors are optimist that the numerical solution by the proposed algorithm can be extended to a wide range i.e., for solving 3D nonlinear time-space fractional order diffusion equations which will be the topic of our future research.

Acknowledgements The authors are extending their heartfelt thanks to the revered reviewers for their valuable comments towards improvement of the quality of the article.

Open Access This article is licensed under a Creative Commons Attribution 4.0 International License, which permits use, sharing, adaptation, distribution and reproduction in any medium or format, as long as you give appropriate credit to the original author(s) and the source, provide a link to the Creative Commons licence, and indicate if changes were made. The images or other third party material in this article are included in the article's Creative Commons licence, unless indicated otherwise in a credit line to the material. If material is not included in the article's Creative Commons licence and your intended use is not permitted by statutory regulation or exceeds the permitted use, you will need to obtain permission directly from the copyright holder. To view a copy of this licence, visit http://creativecommons.org/licenses/by/4.0/.

\section{References}

1. Abdelkawy MA, Taha TM (2014) An operational matrix of fractional derivatives of Laguerre polynomials. Walailak $\mathbf{J}$ Sci Technol 11(12):1041-1055 
2. Abramowitz M, Stegun I (1964) Handbook of mathematical functions dover new york

3. Ateş I, Zegeling P (2017) A homotopy perturbation method for fractional-order advection-diffusion-reaction boundary-value problems. Appl Math Modell 47:425-441

4. Bear J, Verruijt A (1987) Theory and applications of transport in porous media. Reidel, Modeling of groundwater flow and pollution, Dordrecht

5. Ben-Avraham D, Havlin S (2000) Diffusion and reactions in fractals and disordered systems. Cambridge University Press, Cambridge

6. Bhrawy A, Alofi A (2013) The operational matrix of fractional integration for shifted Chebyshev polynomials. Appl Math Lett 26(1):25-31

7. Bhrawy AH, Alghamdi MM, Taha TM (2012) A new modified generalized Laguerre operational matrix of fractional integration for solving fractional differential equations on the half line. Adv Differ Equ 2012(1):179

8. Bouchaud JP, Georges A (1990) Anomalous diffusion in disordered media: statistical mechanisms, models and physical applications. Phys Rep 195(4-5):127-293

9. Brewer J (1978) Kronecker products and matrix calculus in system theory. IEEE Trans Circuits Syst 25(9):772-781

10. Burlayenko VN, Altenbach H, Sadowski T, Dimitrova SD, Bhaskar A (2017) Modelling functionally graded materials in heat transfer and thermal stress analysis by means of graded finite elements. Appl Math Model 45:422-438

11. Chen CM, Liu F, Turner I, Anh V (2010) Numerical schemes and multivariate extrapolation of a two-dimensional anomalous sub-diffusion equation. Numer Algorithms 54(1):1-21

12. Chen S, Liu F, Burrage K (2014) Numerical simulation of a new two-dimensional variable-order fractional percolation equation in non-homogeneous porous media. Comput Math Appl 68(12):2133-2141

13. Chou H, Lee B, Chen C (2006) The transient infiltration process for seepage flow from cracks. Advances in Subsurface Flow and Transport: Eastern and Western Approaches III

14. Das S (2009) A note on fractional diffusion equations. Chaos Solitons Fractals 42(4):2074-2079

15. Das S (2009) Analytical solution of a fractional diffusion equation by variational iteration method. Comput Math Appl 57(3):483-487

16. Dwivedi KD, Das S, Baleanu D et al (2020) Numerical solution of nonlinear space-time fractional-order advectionreaction-diffusion equation. J Comput Nonlinear Dyn 15(6):061005

17. Fomin S, Chugunov V, Hashida T (2011) Mathematical modeling of anomalous diffusion in porous media. Fract Differ Calc 1(1):1-28

18. Gorenflo R, Mainardi F, Moretti D, Pagnini G, Paradisi P (2002) Discrete random walk models for space-time fractional diffusion. Chem Phys 284(1-2):521-541

19. Graham A (2018) Kronecker products and matrix calculus with applications. Courier Dover Publications, New York

20. Guo S, Mei L, Zhang Z, Chen J, He Y, Li Y (2019) Finite difference/Hermite-Galerkin spectral method for multi-dimensional time-fractional nonlinear reaction-diffusion equation in unbounded domains. Appl Math Modell 70:246-263
21. Hashemi MS, Balmeh Z, Baleanu D (2019) Exact solutions, Lie symmetry analysis and conservation laws of the time fractional diffusion-absorption equation. In: Mathematical Methods in Engineering, pp 97-109. Springer

22. He JH (1998) Approximate analytical solution for seepage flow with fractional derivatives in porous media. Comput Methods Appl Mech Eng 167(1-2):57-68

23. Herrmann R (2014) Fractional calculus: an introduction for physicists. World scientific, Singapore

24. Hilfer R (1995) Exact solutions for a class of fractal time random walks. Fractals 3(01):211-216

25. Hilfer R, Anton L (1995) Fractional master equations and fractal time random walks. Phys Rev E 51(2):R848

26. Huang A, et al (1996) A new decomposition for solving percolation equations in porous media. In: Third International Symposium on Aerothermodynamics of Internal Flows, Beijing, China, pp 417-420

27. Jaiswal S, Chopra M, Das S (2019) Numerical solution of non-linear partial differential equation for porous media using operational matrices. Math Comput Simulat 160:138-154

28. Khader M, El Danaf TS, Hendy A (2012) Efficient spectral collocation method for solving multi-term fractional differential equations based on the generalized Laguerre polynomials. Fract Calc Appl 3(13):1-14

29. Kilbas AA, Srivastava HM, Trujillo JJ (2006) Theory and applications of fractional differential equations. Elsevier, Amsterdam

30. Kumar S, Pandey P, Das S, Craciun E (2019) Numerical solution of two dimensional reaction-diffusion equation using operational matrix method based on Genocchi polynomial part I Genocchi polynomial and operational matrix. Proc Rom Acad Ser A Math Phys Tech Sci Inf Sci 20(4):393-399

31. Lewandowski Z, Szynal J (1998) An upper bound for the Laguerre polynomials. J Comput Appl Math 99(1-2):529-533

32. Li X, Wong PJ (2019) Numerical solutions of fourth-order fractional sub-diffusion problems via parametric quintic spline. ZAMM-Journal of Applied Mathematics and Mechanics/Zeitschrift für Angewandte Mathematik und Mechanik p e201800094

33. Magin RL(2006) Fractional calculus in bioengineering, vol. 2. Begell House Redding

34. Mainardi F (2010) Fractional calculus and waves in linear viscoelasticity: an introduction to mathematical models. World Scientific, Singapore

35. Metzler R, Barkai E, Klafter J (1999) Anomalous diffusion and relaxation close to thermal equilibrium: a fractional fokker-planck equation approach. Phys Rev Lett 82(18):3563

36. Metzler R, Klafter J (2000) The random walk's guide to anomalous diffusion: a fractional dynamics approach. Phys Rep 339(1):1-77

37. Moodie T, Tait R (1983) On thermal transients with finite wave speeds. Acta Mech 50(1-2):97-104

38. Nakonieczny K, Sadowski T (2009) Modelling of 'thermal shocks' in composite materials using a meshfree FEM. Comput Mat Sci 44(4):1307-1311 
39. Nepomnyashchy A (2016) Mathematical modelling of subdiffusion-reaction systems. Math Modell Nat Phenomena 11(1):26-36

40. Pandey P, Kumar S, Das S (2019) Approximate analytical solution of coupled fractional order reaction-advectiondiffusion equations. Europ Phys J Plus 134(7):364

41. Podlubny I (1998) Fractional differential equations: an introduction to fractional derivatives, fractional differential equations, to methods of their solution and some of their applications, vol 198. Elsevier, Amsterdam

42. Postek E, Sadowski T (2020) Thermomechanical effects during impact testing of wc/co composite material. Compos Struct, p 112054

43. Povstenko Y (2014) Fundamental solutions to time-fractional advection diffusion equation in a case of two space variables. Math Probl Eng

44. Povstenko Y (2015) Fractional thermoelasticity, vol 219. Springer, Berlin

45. Povstenko Y, Klekot J (2016) The dirichlet problem for the time-fractional advection-diffusion equation in a line segment. Boundary Value Probl 2016(1):89

46. Povstenko Y, Kyrylych T (2017) Two approaches to obtaining the space-time fractional advection-diffusion equation. Entropy 19(7):297

47. Povstenko Y, Kyrylych T (2018) Time-fractional diffusion with mass absorption under harmonic impact. Fract Calc Appl Anal 21(1):118-133

48. Povstenko Y, Kyrylych T, Rygał G (2017) Fractional diffusion in a solid with mass absorption. Entropy 19(5):203

49. Sadowski T (2012) Gradual degradation in two-phase ceramic composites under compression. Comput Mater Sci 64:209-211
50. Sadowski T, Neubrand A (2004) Estimation of the crack length after thermal shock in fgm strip. Int $\mathrm{J}$ Fract 127(2):L135-L140

51. Sadowski T, Pankowski B (2016) Numerical modelling of two-phase ceramic composite response under uniaxial loading. Compos Struct 143:388-394

52. Sadowski T, Samborski S (2003) Prediction of the mechanical behaviour of porous ceramics using mesomechanical modelling. Comput Mater Sci 28(3-4):512-517

53. Sadowski T, Samborski S (2008) Development of damage state in porous ceramics under compression. Comput Mater Sci 43(1):75-81

54. Sadowski T, Hardy SJ, Postek EW (2005) Prediction of the mechanical response of polycrystalline ceramics containing metallic intergranular layers under uniaxial tension. Comput Mat Sci 34(1):46-63

55. Sadowski T, Postek E, Denis Ch (2007) Stress distribution due to discontinuities in polycrystalline ceramics containing metallic inter-granular layers. Comput Mat Sci 39(1):230-236

56. Uchaikin VV (2013) Fractional derivatives for physicists and engineers, vol 2. Springer, Berlin

57. Wu GC, Baleanu D (2013) Variational iteration method for the Burgers' flow with fractional derivatives-new Lagrange multipliers. Appl Math Modell 37(9):6183-6190

Publisher's Note Springer Nature remains neutral with regard to jurisdictional claims in published maps and institutional affiliations. 